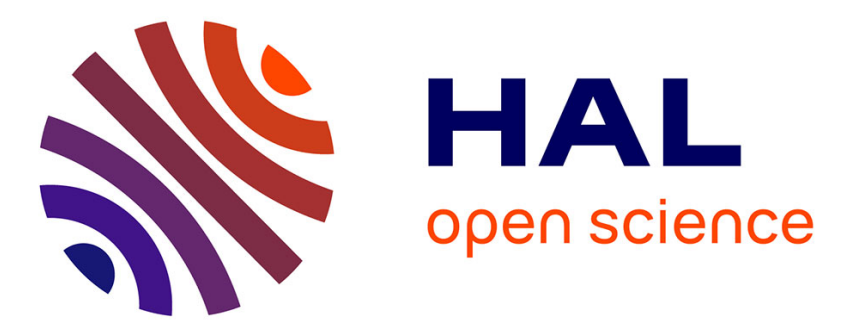

\title{
On the effect of the bending vibration on the acoustic properties of thin poroelastic plates
}

Mark Swift, Kirill Horoshenkov, Philippe Leclaire, David Hothersall, Kyoji Fujiwara, H Torihama

\section{- To cite this version:}

Mark Swift, Kirill Horoshenkov, Philippe Leclaire, David Hothersall, Kyoji Fujiwara, et al.. On the effect of the bending vibration on the acoustic properties of thin poroelastic plates. Journal of the Acoustical Society of America, 2000, 107, 10.1121/1.428577 . hal-01326769

\section{HAL Id: hal-01326769 \\ https://hal.science/hal-01326769}

Submitted on 5 Jun 2016

HAL is a multi-disciplinary open access archive for the deposit and dissemination of scientific research documents, whether they are published or not. The documents may come from teaching and research institutions in France or abroad, or from public or private research centers.
L'archive ouverte pluridisciplinaire HAL, est destinée au dépôt et à la diffusion de documents scientifiques de niveau recherche, publiés ou non, émanant des établissements d'enseignement et de recherche français ou étrangers, des laboratoires publics ou privés. 


\title{
On the effect of the bending vibration on the acoustic properties of thin poroelastic plates
}

\author{
M. J. Swift, K. V. Horoshenkov, P. Leclaire, and D. C. Hothersall \\ Department of Civil and Environmental Engineering, University of Bradford, Bradford BD7 1DP, England \\ K. Fujiwara and $\mathrm{H}$. Torihama \\ Department of Acoustic Design, Kyushu Institute of Design, Shiobaru 4-9-1, Minami, Fukuoka 815, Japan
}

\begin{abstract}
An experimental study on the effect of bending vibration on the acoustic properties of thin porous plates is presented. Poroelastic plates are tested in a large impedance tube in the lower frequency range. It is shown that bending vibration can be excited by the incident sound and detected by measuring the acoustic impedance of a plate. This effect together with the effect of the flow resistivity on the values of the acoustic impedance of the plate are shown. The data from the large and small impedance tubes illustrate how these effects are reduced with the reduced size of the plate.
\end{abstract}

\section{INTRODUCTION}

There are a number of studies which have been carried out on the vibration of plates and structural damping. These have primarily been to investigate behavior of plates which are made from nonporous materials. The results of these studies are widely reported in the literature and a comprehensive summary of them can be found in Ref. 1. On the other hand, some studies have been carried out into the effect of the solid movement on the surface impedance of porous layers.

As an example, particular works by Bardot et al. ${ }^{2}$ and Allard $^{3}$ are concerned with the coupling effect between $\mathrm{Bi}$ ot's slow and fast waves excited in a layer of highly porous fibrous materials with an infinite lateral extend bonded by an impermeable rigid wall. The low rigidity in the thickness of these materials allows compressions and expansions of the porous layer associated with the passage of the airborne acoustic wave. In such a case, the rigid frame approximation where the solid is immobile is no longer valid.

In this article, a coupling between the solid motion and the fluid motion is also studied, but this coupling is of a different nature. Here, the solid movements are eventually taken into account but these correspond to the structural vibration of thin rectangular porous plates. ${ }^{4}$ The same assumption is made as in the standard theory of plates (nonporous) that only the in-plane stresses and deformations are considered while the deformations in the thickness are neglected. This assumption is often written in the standard plate theory as $\sigma_{33}=0$ where $\sigma_{33}$ is the component of the stress tensor normal to the middle surface. As a consequence, the plate is allowed to bend, but its thickness remains constant. Two conditions are required to make this assumption: the plate must be fairly thin and its Young's modulus must be much greater than that of the fluid (air in our case). These requirements are met by our samples.

In this configuration, the triple combination of the bending vibration, the presence of an air gap, and the well-known visco-thermal interaction in the pores are investigated experi- mentally. Their influence on the surface impedance and the absorption coefficient is studied. It is shown that the absorption coefficient of a thin poroelastic plate can be considerably increased at particular frequencies so that the low frequency acoustic performance can be improved.

\section{EXPERIMENTAL PROCEDURE}

An investigation has been carried out on three thin porous plates with varying values of flow resistivity, density, and Young's modulus. Two plates have been manufactured from foam granulates which are recovered from automotive waste. The other plate has been manufactured from flint particles consolidated with rubber-epoxy binder (Coustone ${ }^{\mathrm{TM}}$ ). The dimensions of the plates were $0.5 \mathrm{~m} \times 0.5 \mathrm{~m} \times 10 \mathrm{~mm}$. Other material properties of the plates are listed in Table I.

The measurements have been carried out in the large impedance tube in the Kyushu Institute of Design and in a smaller Bruel and Kjaer 4206 impedance tube available in the University of Bradford. The larger impedance tube allows testing of $0.5 \mathrm{~m} \times 0.5 \mathrm{~m}$ samples of acoustic materials in the frequency range of $50-300 \mathrm{~Hz}$ (see Fig. 1).

The plates have been tested with 40-mm and 80-mm air gaps, depending on the material properties. They have been clamped in the larger impedance tube along their edges using a strong wooden frame and the edges have been carefully sealed (see Fig. 1). Care was taken to ensure good clamping conditions so that not to distort the panel from its "natural" resting position. All gaps between the edges of the frame and the wall of the chamber were blocked using sealing putty. Because of the clamping device, the effective area of the plate exposed to sound was reduced to $0.48 \mathrm{~m} \times 0.48 \mathrm{~m}$.

It is assumed that the resonance frequencies for bending vibration in smaller samples tested in the smaller tube are considerably higher. The results from the normal incidence impedance and absorption from the large and small impedance tubes have been compared. From this comparison the effect of structural vibration on the visco-thermal absorption in the material is estimated. 
TABLE I. Summary table of material properties.

\begin{tabular}{llll}
\hline \hline & \multicolumn{1}{c}{ YB10 } & \multicolumn{1}{c}{ G10 } & \multicolumn{1}{c}{ C10 } \\
\hline Material & Recycled & Recycled & Consolidated \\
& foam & foam & flint \\
Plate thickness (m) & 0.010 & 0.010 & 0.010 \\
Density $\left(\mathrm{kg} / \mathrm{m}^{3}\right)$ & 414 & 390 & 1464 \\
Young's modulus (Pa) & $2.29 \times 10^{7}$ & $1.57 \times 10^{7}$ & $6.20 \times 10^{7}$ \\
Porosity & 0.57 & 0.44 & 0.40 \\
Flow resistivity, Pa s m & & 743200 & 31520 \\
Observed resonance & 190367 & 76.25 & 96.25 \\
frequency, Hz & 86.25 & & \\
Frequency of bending & $89.02(3)$ & $82.54(3)$ & 94.35 (3) \\
wave (mode), Hz & & & \\
\hline
\end{tabular}

Some nonacoustic properties of the tested materials have been measured. These are the bulk density, Young's modulus, porosity, and flow resistivity. The flow resistivity and porosity have been measured using standard techniques of air flow tube and the method of water saturation under vacuum, respectively. The Young's modulus has been measured using rectangular strips of the material in a standard cantilever setup. The values of these parameters are compiled in Table I.

\section{RESULTS AND DISCUSSION}

The results for the real and imaginary parts of the surface acoustic impedance from the measurements in the large and small impedance tubes are compared in Figs. 2-4. The absorption coefficient as a function of frequency is shown in Figs. 5-7.

These figures seem to confirm that the vibration of certain modes in a clamped, porous, elastic plate can be generated by airborne sound in the low frequency range. These modes can be detected as resonances in the acoustic surface impedance (see Figs. 2-4). The frequencies corresponding to the bending vibration modes detected from the measurements are listed in Table I. These frequencies are reasonably close to the theoretical values provided by the classical theory of plates. ${ }^{1}$ At these frequencies the values of the acoustic surface impedance can differ considerably from those measured in the small impedance tube, in which case the amplitude of vibration is considered to be negligibly small (see Figs. 2-4). In the vicinity of these resonances the absolute value of the imaginary part of the impedance can have a minimum, at which the relaxation processes are par-

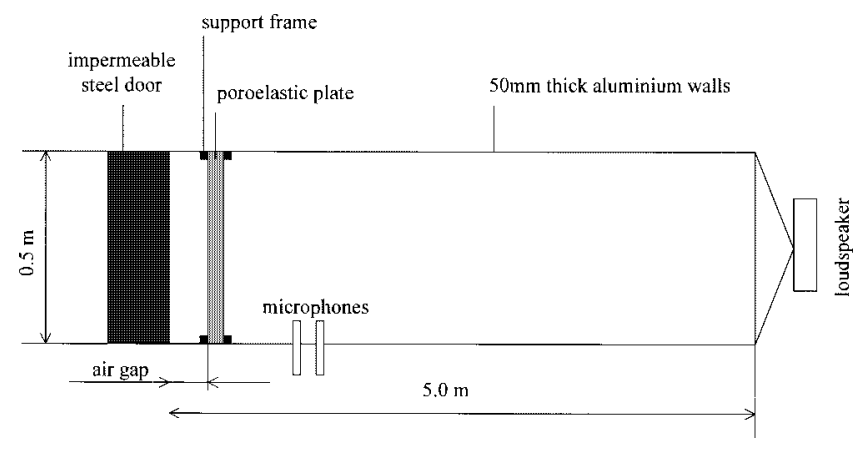

FIG. 1. Experimental setup.

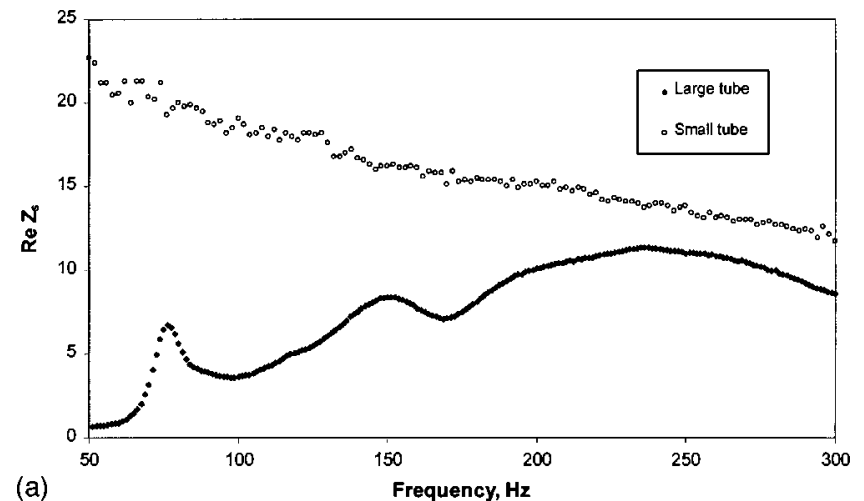

(a)

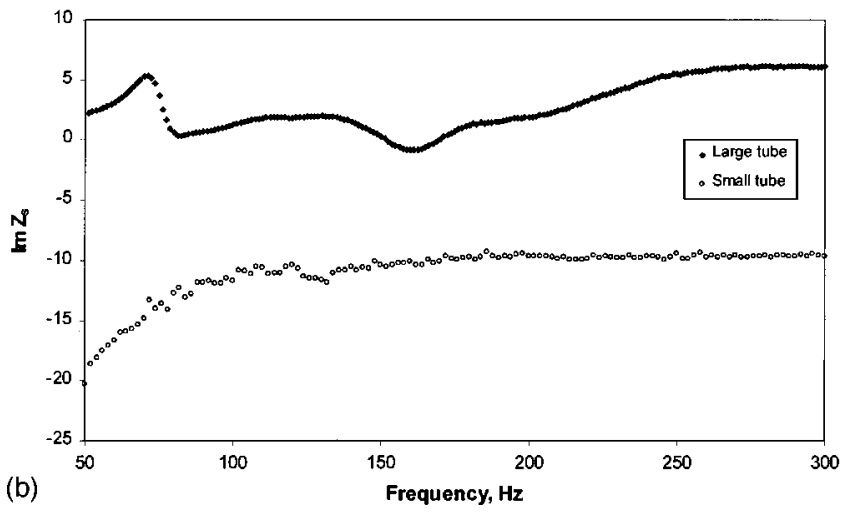

FIG. 2. The real (a) and imaginary (b) parts of the normalized surface impedance of a 10-mm-thick rectangular plate made from consolidated foam G10. 80-mm air gap.

ticularly pronounced. In this region the absorption in the porous material can be noticeably improved (see Figs. 5-7).

In the case of a porous plate which is manufactured from consolidated foam G10 it is possible to observe two bending modes at frequencies $76.25 \mathrm{~Hz}$ and $150 \mathrm{~Hz}$ on the curve for the impedance (see Fig. 2). The frequencies of the modes correspond to the minima in the absorption spectrum (see Fig. 5). The minima in the impedance spectrum for this material are observed at $97.5 \mathrm{~Hz}$ and $168.75 \mathrm{~Hz}$. At these frequencies the absorption is considerably improved in comparison with the data from the small impedance tube (see Fig. 5).

There is a resonance in the impedance spectrum corresponding to a mode which is clearly observed at $86.25 \mathrm{~Hz}$ in the case of a porous plate which is manufactured from consolidated foam YB10 (Fig. 3). There is a local maximum in the absorption spectrum at $76.25 \mathrm{~Hz}$ (Fig. 6).

In the case of the Coustone plate $(\mathrm{C} 10)$ the resonance in the impedance spectrum is observed at $96.25 \mathrm{~Hz}$ (see Fig. 4). There is a local minimum in the spectrum immediately above this frequency, which results in some increase in the absorption spectrum at $103.75 \mathrm{~Hz}$.

A precise quantitative analysis of the problem described in this article is still very difficult at the moment because of the lack of theoretical results. Nevertheless, a few remarks can be made from this study and some conclusions can be drawn with a reasonably good degree of confidence:

(1) The acoustical properties of large acoustic panels measured in a large impedance tube clearly exhibit resonances. These are associated with bending structural vibra- 

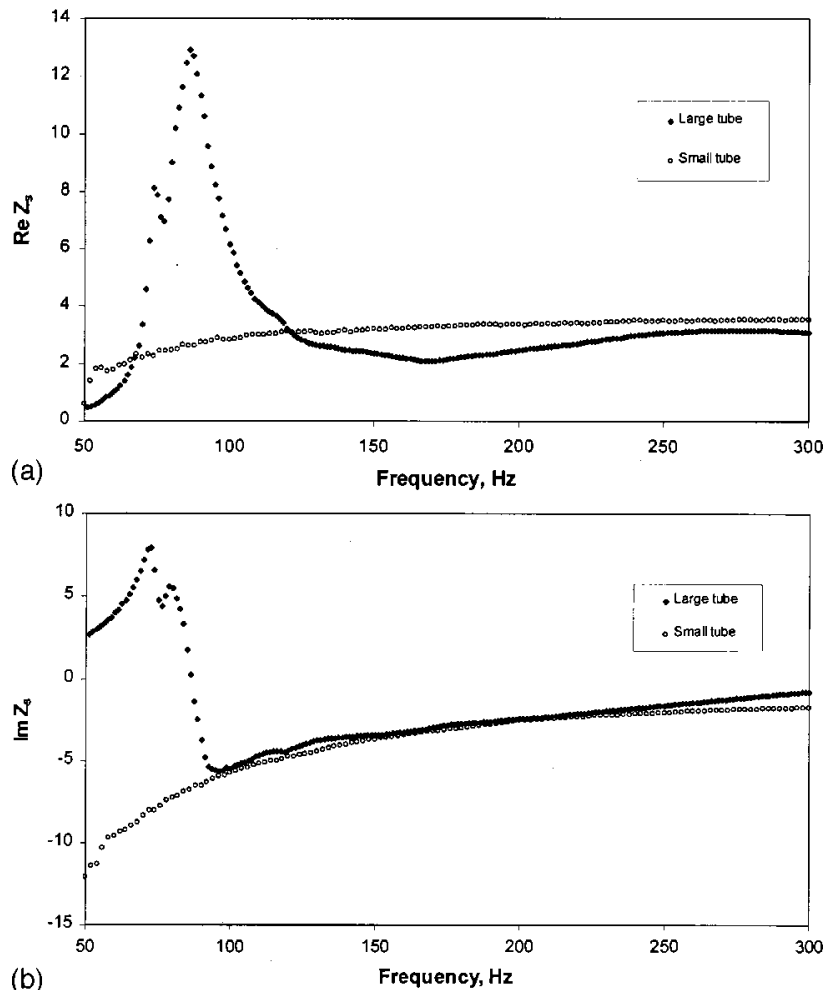

FIG. 3. The real (a) and imaginary (b) parts of the normalized surface impedance of a 10-mm rectangular plate made of consolidated foam YB10. 80-mm air gap.
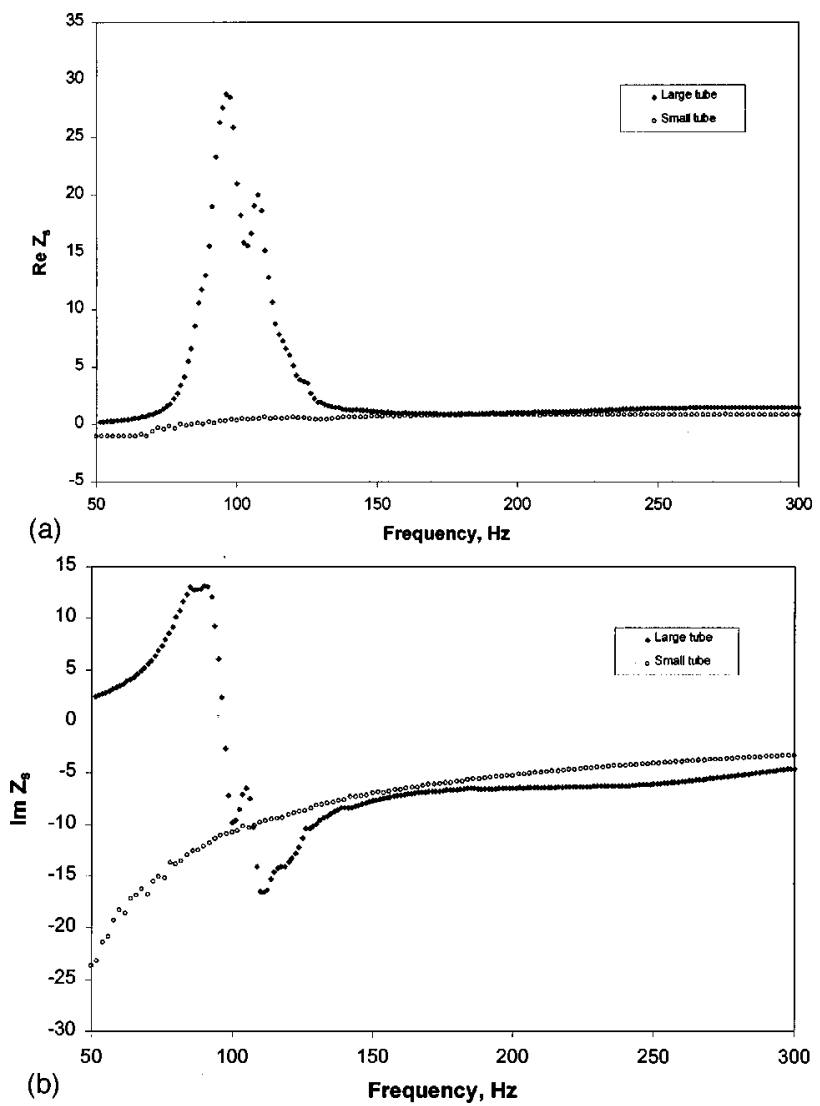

FIG. 4. The real (a) and imaginary (b) parts of the normalized surface impedance of a $10-\mathrm{mm}$ rectangular plate made of the material C10. $40-\mathrm{mm}$ air gap.

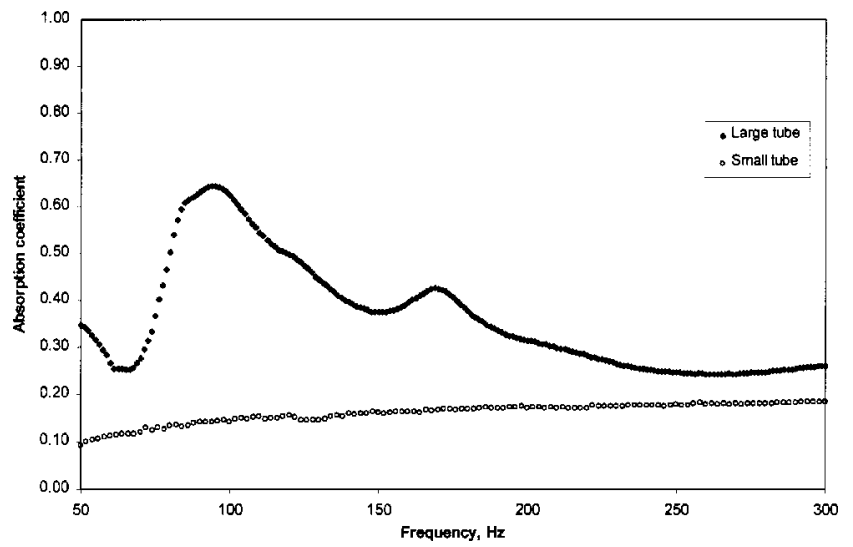

FIG. 5. The normal incidence absorption coefficient of a 10-mm-thick rectangular plate made from consolidated foam G10. 80-mm air gap.

tion of a finite-size, porous plate which has a fairly small thickness and the Young's modulus higher than that of air. The resonance frequencies seem to be close to predictions given by the standard theory of plates. These results show a noticeable enhancement of the absorption coefficient at some frequencies in the low frequency domain where the acoustic absorption is generally low. A physical interpretation of the resonances and of the mechanisms of the enhancement of the absorption coefficient is that a fraction of the acoustic energy carried by the incident sound wave is converted into the vibrational energy used to move the plate. This energy is then partly re-radiated at particular frequencies and partly dissipated in the frame.

(2) It is known that the presence of an air gap with properly chosen dimensions can enhance the low frequency acoustic performances. ${ }^{3}$ It is also known that while increasing the absorption coefficient at some frequency areas, an air gap will decrease it at some others. ${ }^{3}$ Nevertheless, the combination of an air gap with the structural vibrations could be very useful. Depending on its thickness, the presence of an air gap is thought to favor or hinder the observation of the structural vibration, but not to change the resonance frequencies which are solely dependent upon the plate mechanical properties, the clamping conditions, and the microstructural parameters (porosity, tortuosity and flow resistivity).

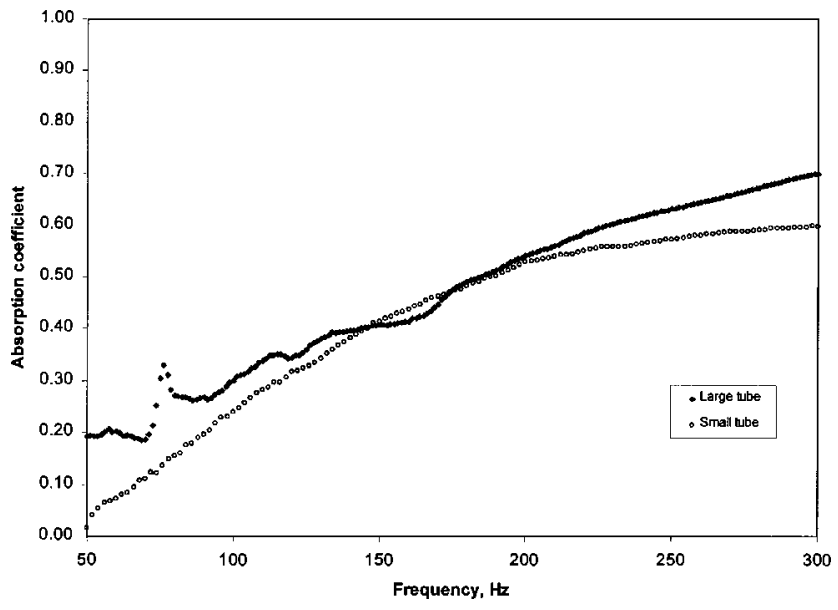

FIG. 6. The normal incidence absorption coefficient of a 10-mm-thick rectangular plate made from consolidated foam YB10. 80-mm air gap. 


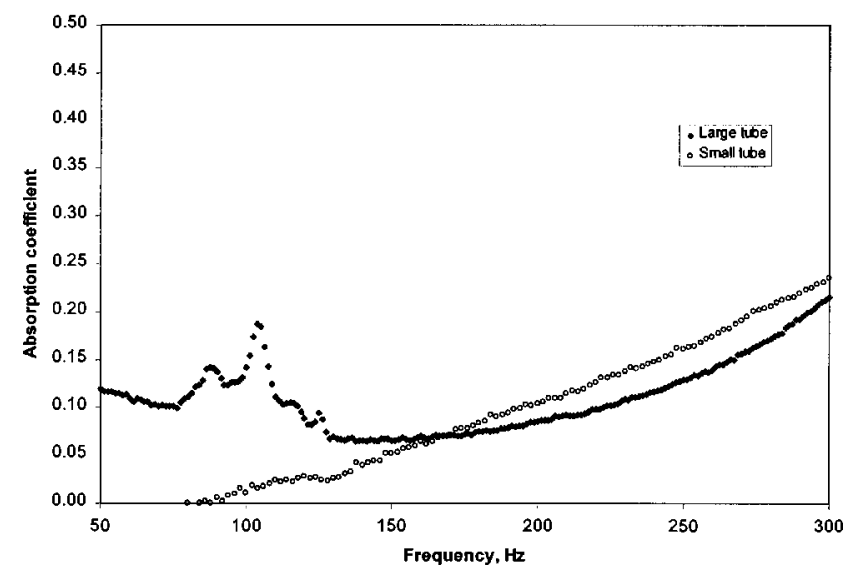

FIG. 7. The normal incidence absorption coefficient of a 10-mm-thick rectangular plate made from Coustone material C10. 40-mm air gap.

(3) The comparison of the damping of the resonances in the three examples investigated in this study shows that peaks in Fig. 5 seem to be smoother than those shown in Figs. 6 and 7. It is thought that particular values of the flow resistivity exist that will result in a maximum loss of energy by viscous frictions at certain frequencies of vibrations of the plate structures. In the optimum case, the phase shift between the solid and fluid motions is maximum and the viscous losses are maximum. This will result in a very smooth resonance peak.

(4) The visco-thermal interactions in porous media have been and are still the subject of active research (see Refs. $3,5)$. At low frequencies, the compressibility of air in the pores is isothermal and the absorption is dominated by the viscous frictions. At higher frequencies the compressibility is adiabatic and the viscous friction is also predominant. The thermal effects are particularly pronounced in the medium frequency range. Although they have less influence on the absorption than the viscous friction, their effect is not negligible. The combination of the visco-thermal effects and the effects described in (1)-(3) can provide an improved absorption spectrum in the whole frequency range.

\section{CONCLUSIONS}

An experimental study has been carried out to determine the effect of bending vibration on the acoustic properties of a clamped, porous, elastic plate. It has been shown that bending vibration of certain modes can be excited by incident sound in the plate and affect its acoustic impedance. The corresponding frequencies can be estimated a using a standard theory for clamped porous plates. ${ }^{1}$ The measured values of the normal incidence plane wave absorption coefficient suggest that, in the vicinity of these frequencies, the absorption can be significantly improved as a result of relaxation processes which develop in the moving porous frame. In this way, porous plates with improved low frequency absorption can be designed. The experimental data also suggest that the acoustic impedance is more affected in porous plates with higher values of the flow resistivity as a result from a better coupling between airborne sound and bending vibration in the elastic frame.

\section{ACKNOWLEDGMENTS}

The authors gratefully acknowledge the support of the Engineering and Physical Sciences Research Council (Grant No. GR/L54905) and the contribution from the British Council in Tokyo toward the travel and expenses connected to the work which was carried out in Kyushu Institute of Design. The authors are also very grateful for the support given by Eric Chadwick and CEP Acoustics Limited, who manufactured and supplied samples of Coustone material.

\footnotetext{
${ }^{1}$ A. Leissa, Vibration of Plates (Acoustical Society of America, New York, 1993).

${ }^{2}$ A. Bardot, B. Brouard, and J. F. Allard, "Frame decoupling at low frequency in thin porous layers saturated by air," J. Appl. Phys. 79, 82238229 (1996).

${ }^{3}$ J.-F. Allard, Propagation of Sound in Porous Media: Modelling Sound Absorbing Materials (Elsevier, Amsterdam, 1993).

${ }^{4}$ D. D. Theodorakopoulos and D. E. Beskos, "Flexural vibrations of poroelastic plates," Acta Mech. 103, 191-203 (1994).

${ }^{5}$ D. Lafarge, P. Lemarinier, J. F. Allard, and V. Tarnow, "Dynamic compressibility of air in porous structures at audible frequencies,' J. Acoust. Soc. Am. 102, 1995-2006 (1997).
} 